\title{
Der zwischensprachliche Transfer beim Drittsprachenerwerb - Einstellungen der DeutschlehrerInnen zum Transfer aus dem Englischen ins Deutsche
}

\section{Interlingual transfer in third language acquisition - German teachers' attitudes towards transfer from English into German}

\begin{abstract}
Interlingual transfer is a well known phenomenon. Thanks to many psycholinguistic studies it is now known which factors can induce or hinder this kind of transfer. In language teaching publications one can find advice on how teachers can deal with interlingual transfer in language acquisition. It is often stressed that transfer can be both negative and positive. The following article presents the results of a survey which was conducted with German teachers from different school types. The aim of the questionnaire was to recognise the teachers' attitudes towards the interlingual transfer from English into German. It turned out that even though a lot of teachers consider such transfer as a negative phenomenon, which should be 'eradicated', some teachers recognise the positive influence of the English language on the acquisition and use of the German language.
\end{abstract}

KEYWORDS: German as a third language, L3, positive and negative interlingual transfer, errors, multicompetence, contrastive analysis.

\section{ZWISCHENSPRACHLICHE EINFLÜSSE}

Zwischensprachliche Einflüsse sind Interaktionen aller Art, die zwischen zwei oder mehreren Sprachen im bilingualen oder multilingualen Kopf stattfinden. Sie entstehen infolgedessen, dass die sich im Gehirn befindenden 
sprachlichen Systeme in denselben neuronalen Arealen gespeichert werden (z.B. Paradis 2004; Perani, Abutalebi 2005). Zu den zwischensprachlichen Einflüssen zählt man: den interlingualen Transfer beim Sprachenlernen, die bewusste Benutzung einer Nicht-Zielsprache in der Kommunikation, Probleme mit dem Abruf von zielsprachlichen lexikalischen Einheiten, die durch eine andere Sprache verursachte Über- oder Unterproduktion einiger zielsprachlicher Elemente, die Verlangsamung der Reaktionszeit, die Sprachabreibung und sogar die Effektivität des Spracherwerbs (Sharwood Smith, Kellerman 1986: 1; Chłopek 2011a: Kap. 4.2).

Die zwischensprachlichen Interaktionen, die im Klassenzimmer am häufigsten zu beobachten sind, sind verschiedene Fälle des interlingualen Transfers während der Produktion und Rezeption. Claus Færch und Gabriele Kasper (1987: 112) definieren den interlingualen Transfer als eine psycholinguistische Prozedur, mithilfe deren L2-Lernende ihre L1/Ln-Kenntnisse aktivieren, um ihre Interimsprache zu entwickeln oder zu benutzen. ${ }^{1}$ Der interlinguale Transfer ist typisch für die Produktion oder Rezeption in einer ungenügend beherrschten Zielsprache und ist eine Art "Rettungstaktik“ des Lernenden, die meistens unbewusst eingesetzt wird. Es werden unterschiedliche Transfererscheinungen identifiziert, wie z.B. lexikalischer Transfer (Kodewechsel, Ad-hoc-Entlehnung, semantische Erweiterung, falsche Freunde, Lehnübertragung/-übersetzung), morphologischer Transfer, orthographischer Transfer, syntaktischer Transfer (syntaktische Lehnübertragung/übersetzung, syntaktisch-semantische Erweiterung) und Transfer der Interpunktion (s. Chłopek 2009, 2011a).

Theoretisch betrachtet können interlinguale Einflüsse zwischen allen sich im Kopf befindenden Sprachen in allen Richtungen verlaufen. Zahlreiche Forschungsstudien, die vor allem mit L3-Lernenden durchgeführt wurden, deuten aber auf bestimmte Faktoren hin, die den zwischensprachlichen Transfer beeinflussen (d. h. beschränken oder hervorrufen) können (z.B. Ringbom 1987; Williams, Hammarberg 1998; Hammarberg 2001, 2009; De Angelis 2005, 2007: 19-40; Gabryś-Barker 2005; Jessner 2008; Jarvis, Pavlenko 2008; s. auch: Cenoz, Jessner 2000; Cenoz, Hufeisen, Jessner 2001; Chłopek 2011a). Die am häufigsten erwähnten Faktoren sind folgende:

- die typologische Verwandschaft der erworbenen und der zu erwerbenden Sprachen - entweder tatsächliche oder perzipierte (Psychotypologie),

- das Kompetenzniveau jeder im Kopf gespeicherten Sprache,

- das Niveau der Aktivierung jeder Sprache,

${ }^{1}$ Im Original: ,"a psycholinguistic procedure by means of which L2 learners activate their L1/Ln knowledge in developing or using their interlanguage“ (Færch, Kasper 1987: 112). 
- die Art und der Kontext des Erwerbs jeder Sprache,

- der Status jeder Sprache (d. h. Muttersprache vs. Fremdsprache - es geht vor allem um den sog. foreign language effect, Meisel 1983),

- die Reihenfolge des Spracherwerbs (der sog. last language effect, Shanon 1991),

- die Funktionen der erworbenen Sprachen.

\section{DER UMGANG MIT DEM ZWISCHENSPRACHLICHEN TRANSFER}

Immer häufiger haben die meisten Fremdsprachenlehrer und -lehrerinnen mit L3-, L4- und sogar L5-Lernenden zu tun. ${ }^{2}$ Je mehr Sprachen zur Verfügung stehen, umso intensiver können die interlingualen Einflüsse sein und umso aufwändiger mag es sein, dass die geeignete und korrekte Auswahl zielsprachlicher Ausdrucksmittel getroffen wird. Wie sollen nun Lehrende mit diesem Phänomen umgehen?

Wie in vielen Publikationen betont wird, dürfen Fremsprachenlehrer und -lehrerinnen nicht so tun, als ob die Lernenden keine anderen Sprachen kannten. Wie aber Ulrike Jessner (1999: 205-206) schreibt, obwohl es für Mehrsprachler ganz natürlich ist, nach den zwischensprachlichen Ähnlichkeiten $\mathrm{zu}$ suchen, werden diese in einem multilingualen Klassenzimmer vernachlässigt. Die übliche Einstellung der Lehrenden ist, das sprachliche Wissen der Lernenden $\mathrm{zu}$ ignorieren oder dieses sogar als eine negative Beeinflussung zu betrachten. ${ }^{3}$

Es ist unmöglich, eine neue (zweite, dritte usw.) Sprache so zu lernen, als ob sie die einzige Sprache im Kopf wäre. L2-/L3-Lernende verfügen über sprachliche und kulturelle Kenntnisse, sowie über Sprachlernerfahrungen, die sich auf den Prozess der Sprachaneignung auswirken. Heutzutage wird es anerkannt,

- dass man, wenn man mehrere Sprachen lernt, nicht jedes Mal sozusagen „bei Null“ anfängt, sondern dass der vorhandene Sprachbesitz durch jede neue Sprache immer mehr erweitert wird,

2 Im weiteren Teil des Textes wird sowohl für die dritten als auch für die weiteren Sprachen das Symbol „L3“ benutzt.

${ }^{3}$ Im Original: "Although the search for commonalities is part of a multilingual person's natural linguistic repertoire, only very few attempts have been made to focus on common elements of the languages in the multilingual classroom. Rather, it seems to be the norm to ignore the prior language knowledge of the students, or, even worse, to regard it as a negative influence" (Jessner 1999: 205-206). 
- dass man nicht in jeder neu zu erlernenden Sprache das Ideal der „nearnativeness" erreichen muss und

- dass das Kompetenzniveau und das Sprachprofil in den einzelnen Sprachen, die man erlernt, sehr unterschiedlich sein kann [...] (Neuner 2003: 16).

Mehrsprachigkeit wird als eine besondere sprachliche Befähigung verstanden, die mit einer "doppelten“ oder "mehrfachen“ Einsprachigkeit nichts zu tun hat (Grosjean 1989). Das sprachliche Wissen einer zwei-/ mehrsprachigen Person - die Multikompetenz (Cook 1991) - ist ein komplexes Wissen, innerhalb dessen sprachliche Systeme miteinander verbunden sind und miteinander interagieren, was zwischensprachliche Einflüsse, wie den interlingualen Transfer, hervorruft. Deswegen ist es für die Lernenden unmöglich, die im Kopf gespeicherten Sprachen voneinander zu trennen, sogar wenn Lehrende ihre Schüler für sie bestrafen. Solche Einstellung der Lehrenden kann den Transfer nicht hindern. Es ist vielmehr sinnvoller, den Lernenden zu ermöglichen, adäquate zwischensprachliche Vernetzungen im Kopf zu bilden. Diese sollten sowohl auf der formalen als auch auf der semantischen Ebene bewusst gebildet werden (s. z.B. Herwig 2001 - Abb. 1). Dank solcher Vernetzungen vermeiden die Lernenden den negativen Transfer und gleichzeitig lernen sie, den positiven Transfer auszunutzen.

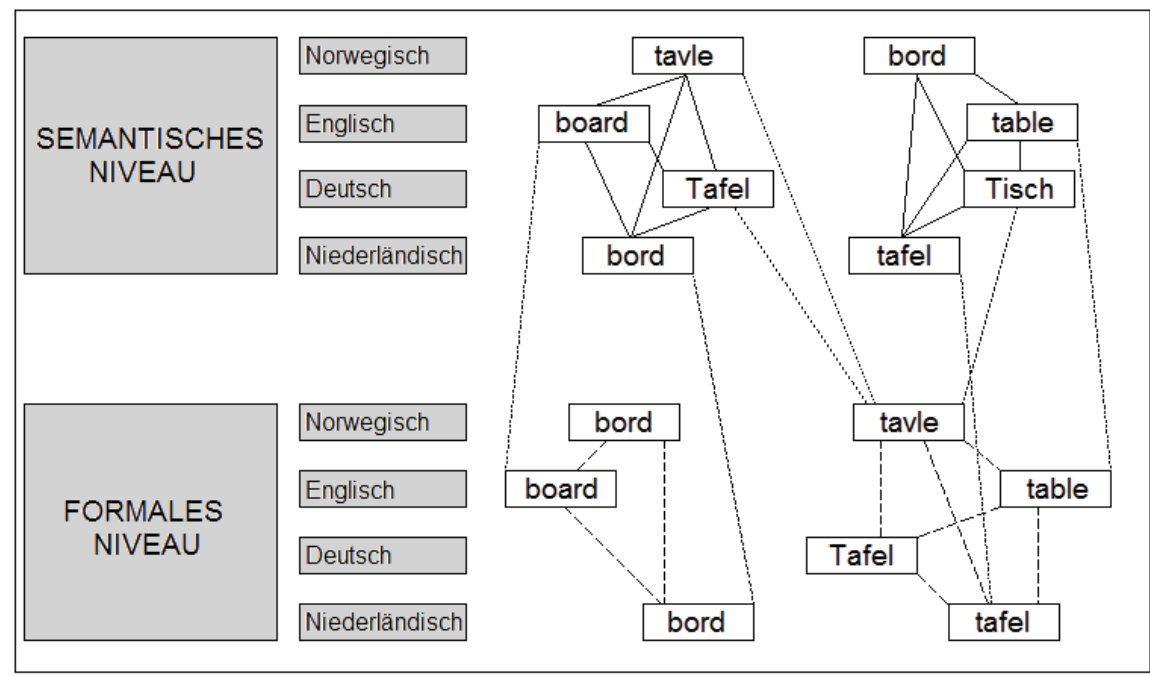

Abb. 1. Symbolische Darstellung des Netzwerkes eines multilingualen mentalen Lexikons nach Anna Herwig (2001)

Das Lehren, das auf den Nicht-Zielsprachen basiert, ist nichts Neues. Es existieren gut bekannte bilinguale Lehrmethoden, wie Dodsons (1967/1972) 
Bilinguale Methode, Currans (1976) Counseling Approach und Lozanovs (1978) Suggestopedie. Auch in der einflussreichen Klassifikation der Lernstrategien von Rebecca L. Oxford (1990) werden einige genannt, die die nicht-zielsprachlichen Kenntnisse der Lernenden zum Einsatz bringen: unter den Kompensationsstrategien erwähnt Oxford den Wechsel in die Muttersprache und unter den kognitiven Strategien erwähnt sie die kontrastive Analyse, die Übersetzung und den Transfer. Viele Forscher (z.B. Meisel 1983; Jessner 1999, 2006; Lewicki 2002; Gibson, Hufeisen 2003; Stopyra 2004) betrachten den bewussten interlingualen Transfer als eine wertvolle kognitive Strategie. Wie Roman Lewicki (2002: 76-77) schreibt: „Die Muttersprache und andere bereits erworbene Fremdsprachen können in vieler Hinsicht als Transferbasis für die Zielsprache dienen und den Erwerb einer neuen Sprache im gesteuerten Lernprozess unterstützen." Es wird oft betont, dass die Berücksichtigung anderer Sprachen im L3-Unterricht den Erwerb einer neuen Sprache erleichtert (Ringbom 1987, 2007; Mißler 1999; Hufeisen, Neuner 2003 [2005]; Hufeisen, Marx 2004; Cummins 2007; Jessner 2008; Kretzenbacher 2009; s. auch Chłopek 2011a: Kap. 3.10).

In Bezug auf den Erwerb einer Zweitsprache (einer ersten Fremdsprache) besteht Wolfgang Butzkamm (2003: 35) auf die Ausnutzung von Parallelen zwischen der Muttersprache und der zu erwerbenden Fremdsprache. Weiterhin betont er, dass die Interferenzfehler nur dann vermieden werden können, wenn die Schüler auf die zwischensprachlichen Unterschiede und Ähnlichkeiten aufmerksam gemacht werden (Butzkamm 2003: 36). Seine Äußerungen können auf die Aneignung einer beliebigen weiteren Fremdsprache verallgemeinert werden. Gerhard Neuner ist der Meinung, die Muttersprache „sollte [...] im Prozess des Erlernens von Fremdsprachen ,im Kopf des Lernenden' nicht ausgeblendet werden, sondern bewusst und aktiv in das Fremdsprachenlernen einbezogen werden, da sie zunächst einmal grundlegend das mentale Sprachennetzwerk strukturiert, in dem alle Elemente, Einheiten und Strukturen der neuen Sprache verankert werden" (Neuner 2003: 19). Die während des muttersprachlichen und zweitsprachlichen Unterrichts entwickelten sprachlichen und kognitiven Fähigkeiten tragen zur Entwicklung der Drittsprache bei (Neuner 2003: 19-24). Eine holistische Einstellung zum Sprachenlehren, die alle Sprachen der Lernenden berücksichtigt, wird auch von der Europäischen Kommission gefördert. Es wird betont, dass solche Politik den Kindern hilft, einen breiten Umfang ihrer kommunikativen Fähigkeiten zu entwickeln. ${ }^{4}$

\footnotetext{
${ }^{4}$ Im Original: „It is important that schools and training institutions adopt a holistic approach to the teaching of language, which makes appropriate connections between the teaching of 'mother tongue', 'foreign' languages, the language of instruction, and the languages of migrant
} 
Es scheint aber, dass das nicht-zielsprachliche Wissen vor allem am Anfang der Sprachaneignung nützlich sein kann, weil es das Verstehen einer neuen Sprache erleichtert und so den Spracherwerb effektiver macht: „Mutter- und fremdsprachliche Kenntnisse in einer oder mehreren Sprachen beschleunigen den Erwerb von rezeptiven Fähigkeiten in der Zielsprache, indem aufgrund der Ähnlichkeiten vorhandenes Wissen aktiviert und zum Verstehen des zielsprachlichen Textes herangezogen werden kann" (Mißler 1999: 45; s. auch Chłopek 2011b).

Es könnte ein Einwand gegen die Einbeziehung von Nicht-Zielsprachen im fremdsprachlichen Unterricht erhoben werden, mit der Begründung, dass je mehr Sprachen aktiviert werden, desto schwieriger mag es sein, alle sprachlichen Systeme im Kopf separat aufzubewahren, und so nur eine Sprache in monolingualen Kontexten problemlos zu verwenden. Wie aber psycholinguistische Forschungsstudien zeigen, weisen L3-Lernende gewisse Merkmale auf, die sie von L2-Lernenden unterscheiden (s. Chłopek 2011a: Kap. 3). Es werden beispielsweise solche Merkmale erwähnt, wie eine hohe metalinguistische Bewusstheit und eine hohe metakognitive Bewusstheit. Die erste ermöglicht $\mathrm{u}$. a. eine bewusste Analyse der Zielsprache und einen Vergleich der schon erworbenen und der zu erwerbenden Sprachen; die zweite bedeutet, dass der Lernprozess bewusst, gezielt und selbstständig stattfindet. Sowohl das komplexe sprachliche Wissen (Multikompetenz) als auch die nicht-sprachlichen Fähigkeiten tragen höchstwahrscheinlich dazu bei, dass L3-Lernende ihre neuen Sprachen schneller und effektiver beherrschen als L2-Lernende (Widła 2007; Chłopek 2011a: Kap. 3.10). In Bezug auf diese Überlegenheit der L3-Lernenden schlägt Britta Hufeisen die Grundprinzipien der Drittsprachendidaktik vor:

Diesen qualitativen Unterschied nun macht sich die Tertiärsprachendidaktik zu Nutze und bezieht explizit die kognitiven und emotionalen Vorerfahrungen der Lernenden im L3-Unterricht mit ein. Wenn die zu lernenden Sprachen sich sehr ähnlich sind, kann dieser Rückgriff sich auch stark auf sprachliche Aspekte beziehen; sind sie eher unterschiedlich, so kommen vielmehr lernstrategische und kognitive Faktoren zum Tragen. Gerade bei diesem Fall ist es auch nicht zwingend notwendig, dass die Lehrkräfte perfekte L2-Sprechende sind. Sie aktivieren nicht in erster Linie die Sprachen selbst, sondern das Lernpotenzial, das beim Lernen dieser vorherigen Sprachen angelegt wurde. So werden Vorerfahrungen nicht länger ignoriert bzw. liegen brach, sondern ermöglichen es, dass der L3-FSU auf einer höheren Stufe beginnen, die Progression steiler angelegt sein und die Inhalte anspruchsvoller sein können (Hufeisen 2003: 9).

communities; such policies will help children to develop the full range of their communicative abilities" (European Commission 2003: 9). 


\section{DIE STUDIE}

Man kann mit Zuversicht behaupten, dass alle Schüler in Polen über einige Kenntnisse des Englischen verfügen. DeutschlehrerInnen haben es also mit Lernenden zu tun, die nicht nur Polnisch (ihre Muttersprache), sondern auch Englisch (und vielleicht andere Fremdsprachen) beherrschen. Mehrere Faktoren können den Transfer zwischen Englisch und Deutsch hervorrufen. Beide Sprachen weisen viele Ähnlichkeiten auf, (psycho-)typologisch also stehen einander nahe, beide werden normalerweise weniger gut beherrscht als die Muttersprache (Polnisch) und beide werden auf ähnliche (gesteuerte) Weise und in ähnlichen (schulischen) Kontexten gelernt. Deutsch und Englisch haben normalerweise den Status einer Fremdsprache, was den foreign language effect hervorrufen kann. Darüber hinaus wird das Deutsche am häufigsten nach dem Englischen gelernt, was den Transfer durch den last language effect auslösen kann.

Einundfünfzig DeutschlehrerInnen in Polen haben einen kurzen Fragebogen ausgefüllt (s. Anhang). ${ }^{5}$ Ziel dieses Fragebogens war es, die Einstellungen der Lehrenden zum Transfer aus dem Englischen ins Deutsche kennen zu lernen. Der Fragebogen setzte sich aus nur 4 Fragen zusammen, um eine hohe Anzahl der RespondentInnen zu sichern. So wurden viele Faktoren (wie z.B. das Alter der LehrerInnen, ihre Sprachkenntnisse und die Sprachkenntnisse ihrer Schüler) außer Betracht gelassen. Die Ergebnisse sind wie folgt:

1. Der Schultyp:

- Grundschule: 8 Personen

- gimnazjum (die im Alter von 13-16 Jahren besuchte Mittelstufenschule): 20 Personen

- szkoła ponadgimnazjalna (eine im Alter von 16-19 Jahren besuchte weitere Schule): 17 Personen

- Hochschule: 10 Personen

- Private Sprachschule: 1 Person

(3 Personen lehren sowohl in gimnazjum als auch in szkoła ponadgimnazjalna. 1 Person lehrt in drei Schultypen: Grundschule, gimnazjum und szkoła ponadgimnazjalna.)

2. In den Aussagen meiner Schüler in der deutschen Sprache beobachte ich den Transfer aus dem Englischen:

- ja, oft: 17 Personen

- ja, manchmal: 27 Personen

- kaum: 6 Personen

- nie: 1 Person

${ }^{5}$ Ich möchte mich an dieser Stelle bei allen Lehrern und Lehrerinnen, die an der Umfrage teilgenommen haben, sehr herzlich bedanken. 
3. Meiner Meinung nach ist dieser Transfer:

- ein durchaus positives Phänomen: 3 Personen

- eher ein positives Phänomen: 18 Personen

- ein durchaus negatives Phänomen: 4 Personen

- eher ein negatives Phänomen: 26 Personen

- ich habe keine Meinung: 2 Personen

(2 Personen haben zwei Möglichkeiten Gewählt: „eher ein positives Phänomen“ und „eher ein negatives Phänomen.")

\section{Zusätzliche Bemerkungen zu Punkt 36:}

1) Dieser Transfer stört die richtige, für die deutsche Sprache relevante Satzstruktur. (Transfer ten zaburza prawidłowa, istotna dla języka niemieckiego budowę zdania.)

2) Wenn man es berücksichtigt, dass Jugendliche schon seit wenigstens 3, manchmal 6 Jahren Englisch lernen, gibt es keine Begründung für die "Verunreiningung" der im gimnazjum eingeführten zweiten Sprache. Dieses Phänomen ist in der Grundschule berechtigt, wo 2 Sprachen gleichzeitig oder mit kleinem Zeitabstand eingeführt werden. (Biorąc pod uwage fakt, że młodzież już co najmniej od 3, nieraz 6 lat uczy się jęz. angielskiego, nie ma powodów, by wprowadzany w gimnazjum drugi jezyk „zanieczyszczony” byt transferami. To zjawisko jest uzasadnione w szkole podstawowej, gdy wprowadzane sa 2 języki w jednym czasie lub w krótkim odstępie czasu.) (Kommentar eines Lehrers/einer Lehrerin aus gimnazjum.)

3) Manche Elemente können aber nützlich sein. (Choć pewne elementy moga być również przydatne.)

4) Die Schüler reagieren auf meine Korrekturen nicht, indem sie kommentieren, dass „sie sich doch auf English auch verständigen werden“. (Uczniowie nie reaguja na poprawę z mojej strony, komentując, że "przecież po angielsku też się dogadaja”.)

5) Der zwischensprachliche Transfer kann als eine Unterstützung für die Veranschaulichung von Ähnlichkeiten und Unterschieden zwischen dem Englischen und dem Deutschen benutzt werden. (Transfer międzyjęzykowy można wykorzystać jako pomoc w pokazaniu podobieństw i różnic między jęz. ang. i jęz. niem.)

6) Eher ein positives Phänomen in erfolgreicher Kommunikation. (Zjawisko raczej pozytywne w skutecznej komunikacji.)

7) Ich habe längere Zeit zwischen eher ein positives und eher ein negatives Phänomen geschwankt. Schließlich habe ich mich für die erste Antwort entschieden, weil in den meisten Fällen bessere Kenntnisse der englischen Sprache (also einer Sprache aus derselben Sprachfamilie!) beim Erraten von Wörtern oder sogar manchen grammatischen Phänomenen helfen. Andererseits aber wenn die Kinder die rote Farbe sehen, sagen sie zuerst "red“, sie denken sogar nicht an das deutsche Wort "rot". (Wahałam się dtuższa chwile między raczej pozytywnym a raczej negatywnym zjawiskiem. Ostatecznie zdecydowałam się na to pierwsze, ponieważ w większości przypadków lepsze obycie z językiem angielskim [językiem z tej samej rodziny językowej!] pomaga w wydedukowaniu stówek czy nawet pewnych zjawisk gramatycznych. Z drugiej jednak strony, kiedy dzieci widza kolor czerwony, najpierw powiedza "red", nie pomyśla nawet o niemieckim "rot".)

8) Die Englischkenntnisse erleichtern den Erwerb mancher deutscher Wörter. (Znajomość angielskiego ułatwia nabywanie niektórych niemieckich słówek.)

${ }^{6}$ Diese Bemerkungen wurden von der Autorin ins Deutsche übersetzt. 
9) Die übertragenen Phänomene (Wörter, Wortfolge, Rechtschreibung) führen häufiger zu Fehlern. Selbst erkennen sie Analogien und Situationen, in denen der Transfer behilflich sein kann, nicht. Ich als Lehrer bin derjenige, der sie auf solche Ähnlichkeiten aufmerksam macht und sie ermutigt, sich solche Phänomene zu merken. (Przenoszone zjawiska [stowa, szyk, ortografia] prowadzą częściej do błędów. Sami nie zauważaja analogii i sytuacji, w których transfer ten byłby pomocny. To raczej ja jako nauczyciel zwracam im uwage na takie podobieństwa $i$ zachęcam do zapamiętywania tych zjawisk.)

10) Als (auch) Psychologe, der sich für die neurobiologische Basis des Spracherwerbs interessiert, weiß ich, dass es ein natürliches Phänomen ist, das von den Prozessen der Verbindung und Trennung der Sprachen im Gehirn zeugt. (Jako [również] psycholog zainteresowany neurobiologicznymi podstawami nabywania jezzyka wiem, że to zjawisko całkowicie naturalne, świadczące o procesach łączenia $i$ rozłączania języków w mózgu.)

11) Abhängig von der eingeführten/geübten Fertigkeit ein positives oder negatives Phänomen - phonetische Übungen, Satzakzent, Grammatik. (W zależności od wprowadzanej/ćwiczonej umiejętności pozytywne lub negatywne - ćwiczenia fonetyczne, akcent $w$ zdaniu, gramatyka.)

12) Bei der Einführung des neuen Materials greife ich oft auf die Ähnlichkeiten mit der englischen Sprache zurück. Der Transfer ist ein negatives Phänomen im Fall eines gleichzeitigen Anfangs des englischen und des deutschen Unterrichts. (Często przy wprowadzaniu nowego materiału odwołuje się do podobieństw w jęz. angielskim. Transfer jest zjawiskiem negatywnym w przypadku, gdybyśmy jednocześnie zaczęli naukę jęz. ang. i jęz. niem.)

13) Dieser Transfer findet vor allem in der schriftlichen Sprache statt. Sie schreiben „ich” mit großem Anfangsbuchstaben, setzen Kommas an seltsamen Stellen, haben auch Probleme mit der Wortfolge. Während des Sprechens begehen sie eher Fehler, die durch mangelnde Sprachkenntnisse verursacht wurden, und nicht weil sie englische Konstruktionen im Kopf haben, wenn sie einen deutschen Satz bilden. (Ten transfer dotyczy przede wszystkim języka pisanego. Pisza "ich" wielka litera, w dziwnych miejscach stawiaja przecinki, maja tez kłopoty z szykiem zdania. $W$ mówieniu to raczej popetniają błędy z niedouczenia, a nie dlatego, że mówiąc zdanie po niemiecku, układaja je, mając na myśli angielskie konstrukcje w głowie.)

14) Der Transfer zeugt von hohem Leistungsniveau in der Ausgangssprache, ich bin aber der Meinung, dass Schüler korrigiert werden und zwischen den Sprachen unterscheiden sollten. (Transfer świadczy o wysokich umiejętnościach językowych języka, z którego on następuje, jednak uważam, że uczniowie powinni być korygowani i rozróżniać między językami.)

4. Wenn dieser Transfer in den Aussagen der Schüler erscheint:

- reagiere ich nicht: 1 Person

- korrigiere ich manchmal, das ist aber von Umständen abhängig: 15 Personen

- versuche ich, immer zu korrigieren: 34 Personen

(1 Person hat einen zusätzlichen Punkt hinzugefügt: "korrigiere ich oft“ ${ }^{\prime}$ )

Zusätzliche Bemerkungen zu Punkt 4:

1) Ich versuche, schlechte Sprachgewohnheiten $\mathrm{zu}$ eliminieren. (Staram się eliminować złe nawyki językowe.) 
2) Ich korrigiere, aber wir suchen nach der Ursache des Transfers zusammen: wir erklären, suchen nach Zusammenhängen. (Poprawiam, ale wspólnie szukamy przyczyny transferu: wyjaśniamy, szukamy powiązań.)

3) Ich korrigiere, indem ich die Schüler auf die zwischensprachlichen Ähnlichkeiten aufmerksam mache, was das Merken erleichtert und den Prozess des Deutschlernens beschleunigt. (Poprawiam, wskazując na podobieństwa między językami, co ułatwia proces zapamiętywania i przyspiesza proces uczenia się jęz. niemieckiego.)

4) Ich korrigiere während der Spiele nicht. (Nie poprawiam w trakcie zabaw.)

5) Immer mache ich sie auf die Quelle eines solchen Fehlers aufmerksam. (Zawsze zwracam im uwagę na źródto takiego błędu.)

6) Am häufigsten betrifft der Transfer die Syntax. (Najczęściej dotyczy składni.)

7) Oft bin ich nicht imstande zu erkennen, dass die Konstruktion, auf der die Kursteilnehmer "bestehen“, eine Lehnübersetzung aus dem Englischen ist, obwohl meine rezeptiven Kenntnisse im Englischen auf dem Niveau B2/C1, aber meine produktiven Kenntnisse auf dem Niveau A2/B1 sind. (Często nie jestem w stanie wytapać, że konstrukcja, przy której „upierają się" kursanci, jest kalką z jęz. angielskiego, mimo że umiejętności rozumienia angielskiego mam na poziomie B2/C1, ale umiejętności produktywne na poziomie A2/B1.)

8) Ich korrigiere, indem ich das deutsche Äquivalent nenne. (Poprawiam, podajac niemiecki odpowiednik.)

9) Ich nutze die Englischkenntnisse meiner Studenten aus, damit ich manche englische Elemente in die von mir gelehrte deutsche Sprache übertrage oder auf die Kontraste deutlich hinweise. (Wykorzystuje znajomość jęz. angielskiego u studentów, żeby przenieść niektóre jego elementy do nauczanego przeze mnie jęz. niemieckiego lub téz wyraźnie wskazać na kontrasty.)

10) Ich thematisiere es, um die Wichtigkeit dieses Themas im Sprachunterricht bewusst zu machen (Aussage auf Deutsch).

11) Ich korrigiere dann, wenn der Anglizismus in das deutsche Lexikon noch nicht aufgenommen wurde. (Poprawiam wtedy, gdy dany anglicyzm nie zostat jeszcze przyjęty do niemieckiego stownictwa.)

Die meisten DeutschlehrerInnen haben den Transfer aus dem Englischen beobachtet - entweder oft (17 Personen) oder manchmal (27 Personen). Höchstwahrscheinlich hat es mehr Transfer gegeben als beobachtet wurde LehrerInnen erkennen bestimmt nicht jeden Fall des Transfers (s. auch Bemerkung 7 zum 4. Punkt).

Wie perzipieren die RespondentInnen diesen Transfer? Für 21 Personen ist er ein positives Phänomen (entweder ein durchaus positives Phänomen 3 Personen, oder eher ein positives Phänomen - 18 Personen); für 30 Personen ist er ein negatives Phänomen (entweder ein durchaus negatives Phänomen - 4 Personen, oder eher ein negatives Phänomen - 26 Personen) (2 Personen haben zwei Möglichkeiten gewählt: „eher ein positives Phänomen" und "eher ein negatives Phänomen"); zwei Personen haben keine Meinung. Die Mehrheit also betrachtet den zwischensprachlichen Transfer als etwas Unerwünschtes. Ich bin überzeugt, dass diese LehrerInnen den 
positiven Transfer und seine Auswirkung auf das Tempo und die Effektivität des Spracherwerbs nicht erkannt haben. Ein Grund für eine solche Einstellung könnten die unausreichenden Englischkenntnisse der RespondentInnen sein. Ein anderer Grund könnte eine bestimmte Geschlossenheit der LehrerInnen auf den Spracherwerbsprozess und seine Komplexität sein. Darüber hinaus wissen manche LehrerInnen vielleicht einfach nicht, dass der zwischensprachliche Transfer nicht nur negativ, sondern auch positiv sein kann; deswegen unterschätzen sie das Potenzial des Transfers.

Andererseits schätzen ziemlich viele LehrerInnen den positiven Einfluss des Englischen auf den Erwerb des Deutschen richtig ein und wissen ihn auszunutzen. In ihren zusätzlichen Bemerkungen zum 3. Punkt haben sich 8 LehrerInnen zu dem (teilweise) positiven Einfluss des Englischen geäußert (Bemerkungen 3, 5, 6, 7, 8, 9, 11, 12). Aus einigen Kommentaren wird ersichtlich, dass manche LehrerInnen den interlingualen Transfer als sowohl nützlich als auch störend für die Entwicklung des Deutschen betrachten $(3,7,8$, 9, 11, 12). Sie bemerken also die negative Auswirkung des Englischen und gleichzeitig verstehen sie das Potential seiner Nutzung während des Unterrichts.

Mit Ausnahme einer Person (die auch keine Transferfehler beobachtete), korrigieren alle LehrerInnen die Transferfehler aus dem Englischen. Die meisten (34 Personen) versuchen, sie immer zu korrigieren. Dieses Ergebnis mag davon zeugen, dass manche LehrerInnen die Natur der Multikompetenz nicht verstehen. Wie oben erwähnt, ist die multilinguale Kompetenz keine einfache Summe der monolingualen Kompetenzen. Es ist unmöglich, eine neue Sprache genauso wie die Erstsprache - also wie die einzige Sprache - zu erlernen und sie im Kopf separat aufzubewahren. Infolgedessen engagieren Mehrsprachler alle ihre Sprachen während der Kommunikation, wenn es auch zum großen Teil unbewusst verläuft und von ihren Gesprächspartnern nicht erkannt wird. Deswegen, wenn man seine dritte oder weitere Sprache erlernt, soll das Hauptziel des Lernprozesses die erfolgreiche Kommunikation in echten Situationen des Alltags sein, die unterschiedliche Strategien, darunter den zwischensprachlichen Transfer, einbezieht. Obwohl diese Fehler, die die Kommunikation stören, natürlich sofort korrigiert werden sollten, müsste man die Transfererscheinungen, die (besonders während freier Produktion) eine kompensatorische Funktion ausüben, die also von erfolgreicher Ausnutzung der Multikompetenz zeugen, als ein Vorteil des L3-Lernenden betrachtet werden.

Manche LehrerInnen können auch davon ausgehen, dass ihre Englischkenntnisse unausreichend sind, um die englische Sprache in den Deutschunterricht einzuführen. Man muss aber über keine perfekten Kompetenzen verfügen, um die Schüler auf einige wichtige interlinguale Ähnlichkeiten 
und Unterschiede aufmerksam zu machen. Eine gute Lösung scheint die $\mathrm{Zu}$ sammenarbeit zwischen Deutschlehrenden und Englischlehrenden zu sein, infolge deren zwischensprachliche Übungen zusammen entwickelt werden können.

Andererseits zeigen einige Bemerkungen zum 3. Punkt $(5,9,12)$ und zum 4 . Punkt $(2,3,5,9)$, dass manche LehrerInnen verstehen, dass die Korrektur in Form einer kontrastiven Analyse durchgeführt werden kann, die die zwischensprachlichen Unterschiede und Ähnlichkeiten verstehen lässt und so für den Erwerb des Deutschen behilflich sein kann, indem Lernende den negativen Transfer vermeiden und den positiven Transfer ausnutzen.

Die Zahl der RespondentInnen ist leider nicht groß genug, um zu erkennen, ob Lehrende in verschiedenen Schultypen unterschiedliche Einstellungen zum Transfer haben. Eine interessante Tendenz, die aber noch geprüft werden sollte, ist die Neigung der Hochschullehrenden, die Transferfehler nur unter Umständen zu korrigieren: unter den 10 Hochschullehrenden haben sogar 7 RespondentInnen diese Antwort gewählt, und unter den 41 Lehrenden aus anderen Schultypen haben sich nur 8 RespondentInnen für diese Antwort entschieden; die meisten Nicht-Hochschullehrenden versuchen, die Transfererscheinungen immer zu korrigieren.

\section{ZUSAMMENFASSUNG}

Die Resultate dieser Studie zeigen, dass der Transfer aus dem Englischen während des Erwerbs des Deutschen ein weit verbreitetes Phänomen ist. Gleichzeitig lassen sie vermuten, dass DeutschlehrerInnen den positiven zwischensprachlichen Transfer oft nicht erkennen. Infolgedessen betrachten sie ihn oft als ein ausschließlich negatives Phänomen, das den Deutscherwerb hindern kann und deshalb „ausgerottet“ werden soll.

Die gewonnenen Resultate lassen auch vermuten, dass viele Lehrende die Natur der Multikompetenz nicht verstehen. Deswegen wissen sie nicht, dass der Transfer einerseits unvermeidlich ist (auch wenn andere Sprachen nicht aktiviert werden), andererseits behilflich sein kann. So weigern sie sich, andere Sprachen der Lernenden in den Lehrprozess einzubeziehen.

Manche LehrerInnen aber verstehen den Vorteil der kontrastiven Analyse während des Unterrichts und geben sich Mühe, ihren SchülerInnen zu ermöglichen, sich auf ihre komplexen Sprachkompetenzen zu stützen, d. h. von allen ihren Sprachen sinnvollen Gebrauch zu machen, damit sie Transferfehler vermeiden und aus zwischensprachlichen Ähnlichkeiten schöpfen können. 
Zum Schluss sollte noch einmal betont werden, dass gute Englischkenntnisse keine Voraussetzung für einen erfolgreichen Deutschunterricht sind. Alle Deutschlehrenden ohne spezielle Erfahrungen können eine informelle kontrastive Analyse für ihre praktischen Unterrichtszwecke durchführen und die Ergebnisse mit ihren Englisch unterrichtenden Kollegen durchdiskutieren.

\section{LITERATURVERZEICHNIS}

Butzkamm, W., 2003. We only learn language once. The role of the mother tongue in FL classrooms: death of a dogma. In: Language Learning Journal, 28, 29-39.

Cenoz, J., Hufeisen, B., Jessner, U. (Hrsg.), 2001. Cross-linguistic Influence in Third Language Acquisition: Psycholinguistic Perspectives. Clevedon: Multilingual Matters.

Cenoz, J., Jessner, U. (Hrsg.), 2000. English in Europe: The Acquisition of a Third Language. Clevedon: Multilingual Matters.

Chłopek, Z., 2009. Transferfehler in der schriftlichen Produktion der polnischen Germanistikstudenten in ihrer Drittsprache - Englisch: Zum Form- und Inhaltstransfer. In: Kunicki W., Rzeszotnik J., Tomiczek E. (Hrsg.). Breslau und die Welt: Festschrift für Prof. Dr. Irena Świattowska-Prędota zum 65. Geburtstag. Wrocław, Dresden: Oficyna Wydawnicza ATUT - Wrocławskie Wydawnictwo Oświatowe, Neisse Verlag, 515529.

Chłopek, Z., 2011a. Nabywanie języków trzecich i kolejnych oraz wielojęzyczność: Aspekty psycholingwistyczne ( $i$ inne). Wrocław: Wydawnictwo Uniwersytetu Wrocławskiego.

Chłopek, Z., 2011b. Das Lehren von Deutsch als Drittsprache in Polen: Methodologische Hinweise. In: Białek E., Huszcza K., Lipiński C. (Hrsg.). Texte in Bewegung setzen: Aufsätze zur Literatur- und Fremdsprachendidaktik. Dresden, Wrocław: Neisse Verlag, Quaestio, 127-140.

Ciekanski, M., 2005. Towards the development of a plurilingual and pluricultural competence. In: Preisler B., Fabricius A., Haberland H., Kjærbeck S., Risager K. (Hrsg.). The Consequences of Mobility. Roskilde: Roskilde University, Department of Language and Culture, 163-170.

Cook, V.J., 1991. The poverty of the stimulus argument and multicompetence. In: Second Language Research, 7/2, 103-117.

Cummins, J., 2007. Rethinking monolingual instructional strategies in multilingual classrooms. In: Canadian Journal of Applied Linguistics/Revue canadienne de linguistique appliqué, 10/2, 221-240.

Curran, C.A., 1976. Counseling-Learning in Second Languages. Apple River, IL: Apple River Press.

De Angelis, G., 2005. Multilingualism and non-native lexical transfer: an identification problem. In: International Journal of Multilingualism, 2/1, 1-25.

De Angelis, G., 2007. Third or Additional Language Acquisition. Clevedon: Multilingual Matters.

Dodson, C.J., 1967/1972. Language Teaching and the Bilingual Method. London: Pitman.

European Commission. 2003. Communication from the Commission to the Council, the European Parliament, the Economic and Social Committee and the Committee of the Regions. Promoting Language Learning and Linguistic Diversity: An Action Plan 2004-2006. 
<http:/ / eur-lex.europa.eu/legal-content/EN/TXT/?uri=CELEX:52003DC0449>, [abgerufen am 30.01.2015].

Færch, C., Kasper, G., 1987. Perspectives on language transfer. In: Applied Linguistics, 8/2, 111-136.

Gabryś-Barker, D., 2005. Aspects of Multilingual Storage, Processing and Retrieval. Katowice: Wydawnictwo Uniwersytetu Śląskiego.

Gibson, M., Hufeisen, B., 2003. Investigating the role of prior foreign language knowledge: translating from an unknown into a known foreign language. In: Cenoz J., Hufeisen B., Jessner U. (Hrsg.). The Multilingual Lexicon. Dordrecht, Boston, London: Kluwer Academic Publishers, 87-102.

Grosjean, F., 1989. Neurolinguists, beware! The bilingual is not two monolinguals in one person. In: Brain and Language, 36/1, 3-15.

Hammarberg, B., 2001. Roles of L1 and L2 in L3 production and acquisition. In: Cenoz J., Hufeisen B., Jessner U. (Hrsg.). Cross-linguistic Influence in Third Language Acquisition: Psycholinguistic Perspectives. Clevedon: Multilingual Matters, 21-41.

Hammarberg, B. (Hrsg.), 2009. Processes in Third Language Acquisition. Edinburgh: Edinburgh University Press.

Herwig, A., 2001. Plurilingual lexical organisation: Evidence from lexical processing in L1-L2-L3-L4 translation. In: Cenoz J., Hufeisen B., Jessner U. (Hrsg.). Cross-linguistic Influence in Third Language Acquisition: Psycholinguistic Perspectives. Clevedon: Multilingual Matters, 115-137.

Hufeisen, B., 2003. Kurze Einführung in die linguistische Basis. In: Hufeisen B., Neuner G. (Hrsg.). Mehrsprachigkeitskonzept - Tertiärsprachenlernen - Deutsch nach Englisch. Strasbourg, Europarat: Council of Europe Publishing, 7-11.

Hufeisen, B., Marx, N. (Hrsg.), 2004. Beim Schwedischlernen sind Englisch und Deutsch ganz hilfsvoll. Untersuchungen zum multiplen Sprachenlernen. Frankfurt/M: Peter Lang.

Hufeisen, B., Neuner G. (Hrsg.), 2003 [2005]. Mehrsprachigkeitskonzept - Tertiärsprachenlernen - Deutsch nach Englisch. Strasbourg, Europarat: Council of Europe Publishing [2. Auflage].

Jarvis, S., Pavlenko, A., 2008. Crosslinguistic Influence in Language and Cognition. New York, Abingdon: Routledge.

Jessner, U., 1999. Metalinguistic Awareness in Multilinguals: Cognitive Aspects of Third Language Learning. In: Language Awareness, 8/3 \& 4, 201-209.

Jessner, U., 2006. Linguistic Awareness in Multilinguals: English as a Third Language. Edinburgh: Edinburgh University Press.

Jessner, U., 2008. Teaching third languages: Findings, trends and challenges. In: Language Teaching, 41/1, 15-56.

Kretzenbacher, H.L., 2009. Deutsch nach Englisch: Didaktische Brücken für syntaktische Klammern. In: Electronic Journal of Foreign Language Teaching, 6(1), 88-99.

Lewicki, R., 2002. Guten Tag. Wie geht's? Dziękuję, wszystko w porządku. Zur Entwicklung der Rezeptiven bilingualen Kompetenz. In: Neveling C. (Hrsg.). Perspektiven für die zukünftige Fremdsprachendidaktik, Tübingen. Gunter Narr Verlag, 75-88.

Lozanov, G., 1978. Suggestology and Outlines of Suggestopedy. New York: Gordon and Breach

Meisel, J.M., 1983. Transfer as a second language strategy. In: Language and Communication, 3/1, 11-46.

Mißler, B., 1999. Fremdsprachenlernerfahrungen und Lernstrategien. Eine empirische Untersuchung. Tübingen: Stauffenburg. 
Neuner, G., 2003 [2005]. Mehrsprachigkeitskonzept und Tertiärsprachendidaktik. In: Hufeisen B., Neuner G. (Hrsg.). Mehrsprachigkeitskonzept - Tertiärsprachenlernen Deutsch nach Englisch. Strasbourg, Europarat: Council of Europe Publishing, 13-34.

Oxford, R.L., 1990. Language Learning Strategies. What Every Teacher Should Know. Boston, MA: Heinle \& Heinle.

Paradis, M., 2004. A Neurolinguistic Theory of Bilingualism. Amsterdam, Philadelphia, PA: John Benjamins.

Perani, D., Abutalebi, J., 2005. The neural basis of first and second language processing. In: Current Opinion in Neurobiology, 15/2, 202-206.

Ringbom, H., 1987. The Role of the First Language in Foreign Language Learning. Clevedon: Multilingual Matters.

Ringbom, H., 2007. Cross-linguistic Similarity in Foreign Language Learning. Clevedon, Buffalo, Toronto: Multilingual Matters.

Shanon, B., 1991. Faulty Language Selection in Polyglots. In: Language and Cognitive Processes, 6/4, 339-350.

Sharwood Smith, M., Kellerman, E., 1986. Crosslinguistic influence in second language acquisition: an introduction. In: Kellerman E., Sharwood Smith M. (Hrsg.), Crosslinguistic Influence in Second Language Acquisition. Oxford: Pergamon, 1-9.

Stopyra, J., 2004. Über das Deutsche zum Dänischen: Ein Umweg für polnische Germanistikstudenten mit Dänisch als zweiter Fremdsprache. In: ORBIS LINGUARUM, 27, 305-308.

Widła, H., 2007. L'acquisition du français - langue troisième: Problèmes méthodologiques et implications pratiques. Katowice: Wydawnictwo Uniwersytetu Śląskiego.

Williams, S., Hammarberg, B., 1998. Language switches in L3 production: Implications for a polyglot speaking model. In: Applied Linguistics, 19/3, 295-333. 


\section{Anhang - Fragebogen für Deutschlehrende}

Ankieta dla nauczycieli języka niemieckiego

dotycząca transferu z języka angielskiego na język niemiecki

w wypowiedziach uczniów

1. Typ szkoły (podstawowa, gimnazjum, ponadgimnazjalna itp.):

2. W wypowiedziach moich uczniów w jęz. niemieckim obserwuję transfer z jęz. angielskiego:

$\square$ tak, często $\square$ tak, czasami $\square$ rzadko kiedy $\square$ nigdy

3. Uważam, że ten transfer to:

$\square$ zjawisko zdecydowanie pozytywne

$\square$ zjawisko raczej pozytywne

$\square$ zjawisko zdecydowanie negatywne

$\square$ zjawisko raczej negatywne

$\square$ nie mam zdania

Dodatkowe uwagi:

4. Gdy taki transfer pojawia się w wypowiedziach uczniów:

$\square$ Nie reaguję na niego.

$\square$ Czasem poprawiam, ale to zależy.

$\square$ Zawsze staram się poprawiać.

Dodatkowe uwagi:

Bardzo dziękuję za wypełnienie ankiety! :)

W razie pytań proszę o kontakt pod adresem: chlopekzofia@gmail.com 